\title{
Combined thrombolytic and endovascular treatment of proximal deep vein thrombosis in inferior vena cava agenesis
}

\author{
Boštjan Leskovar ${ }^{1}$, Tjaša Furlan ${ }^{1 *}$ and Milenko Stanković ${ }^{2}$ \\ ${ }^{1}$ Department of Internal Medicine, Trbovlje General Hospital, Slovenia \\ ${ }^{2}$ University Medical Centre Ljubljana, Clinical Institute of Radiology, Slovenia
}

\begin{abstract}
A 20-year-old female was admitted to our hospital because of a swollen left leg and chest pain. Doppler ultrasound revealed deep venous thrombosis of calf and femoral veins. Despite therapeutic doses of low molecular weight heparin, the thrombosis progressed to iliac region. Regional thrombolysis was only partially successful. Abdominal CTA showed inferior vena cava agenesis. We continued the treatment with a combination of stepped endovascular treatment and low dose regional thrombolysis. Complete re-establishment of the venous flow in the left lower limb, left iliac veins, left paravertebral veins, azygos and hemiazygos vein was observed after 14 days of treatment. After two years, she is on long-term oral anticoagulant therapy and the previously thrombosed venous system is still completely patent.
\end{abstract}

\section{Introduction}

Inferior vena cava agenesis (IVCA) is a rare developmental abnormality [1] which most commonly presents with deep venous thrombosis (DVT) in patients aged 20-30 years, more commonly in men [1-5]. Less than 100 cases of patients with atresia/agenesis of inferior vena cava are described in the literature [5]. There are no guidelines for treatment of DVT in these patients, studies report anticoagulant treatment, thrombolysis and even surgical bypass [4-6].

\section{Case report}

A 20-year-old female with no known comorbidities presented to our hospital with left lower limb swelling and chest pain. Doppler ultrasound confirmed DVT of superficial femoral vein, non-occlusive thrombosis of common femoral vein on the left leg and thrombosis of calf veins. After excluding pregnancy and other contraindications for contrast CT, we decided to do a CTA of the pulmonary arteries, which excluded acute pulmonary embolism. Transthoracic echocardiography showed normal cardiac anatomy and function. Low molecular weight heparin (LMWH) in therapeutic dosage was started and she was discharged home in stable condition after three days. Ten days later she was readmitted due to increasing left leg swelling despite treatment with therapeutic doses of LMWH. Doppler ultrasound showed thrombus progression: we confirmed occlusive thrombosis of the left iliac veins, all left lower limb deep veins, the small saphenous vein (SSV) and saphenofemoral junction (SFJ). Left great saphenous vein (GSV) was patent and it drained to perineal area. Abdominal ultrasound and gynaecological examination were done to exclude pelvic and paraaortic masses. We decided to start with local thrombolysis, according to our protocol. The protocol has been created and modified in the last 15 years based on our experience with thrombolysis of haemodialysis vascular access. It is based on minimal systemic and large local effect of recombinant tissue plasminogen activator ( $r$-tPA) (80\% of effect is diminished in 10 minutes). Low dose thrombolysis $(0.2-1 \mathrm{mg} / \mathrm{h})$ is applied through a catheter inserted into or near the thrombus. Simultaneously with thrombolysis, a heparin infusion is added for the prevention of systemic embolism (aPTT 1.5-2 times the baseline value) and a constant infusion of normal saline $(100 \mathrm{ml} / \mathrm{h})$ assuring constant flow. Duration of thrombolysis is defined by its success (monitored with Doppler ultrasound and/or angiographically) and haemostasis tests (aPTT, TT, fibrinogen, d-dimer). We observed high success rate and less than $2.5 \%$ of clinically important complications with our thrombolysis protocol in treating haemodialysis vascular access thrombosis. Our protocol is like Baekgaard's which showed good longterm results in patients with ileofemoral thrombosis [7]. Both protocols were developed simultaneously and independently.

This time we used this protocol for DVT thrombolysis. A 4 Fr sheath was introduced under ultrasound guidance anterogradelly into the upper part of the left GSV. Then a 4 Fr catheter was placed into the occluded part of the left internal iliac vein (proximally to the sheath). We did not decide to puncture the thrombosed popliteal vein because this would be more difficult and risky than puncturing the superficial GSV. Technically, the puncture of occluded popliteal vein is difficult because of its anatomical position and its vicinity to popliteal artery. In case of unsuccessful venous puncture or incidental arterial function, thrombolysis would be contraindicated. Also, the primary

Correspondence to: Tjaša Furlan, Department of Internal Medicine, Trbovlje General Hospital, Rudarska cesta 91420 Trbovlje Slovenia, Tel: 0038635652679; Fax: 003863562612; E-mail:tjasa.furlan@gmail.com

Key words: deep vein thrombosis, inferior vena cava agenesis, thrombolysis, endovascular treatment

Received: November 16, 2017; Accepted: December 07, 2017; Published: December 11, 2017 
goal of thrombolysis was not to dissolve the thrombus completely, but to reduce the extent of thrombosis from class I-IV to class I-II (LET - lower extremity thrombosis classification), which has significantly lower incidence of post-thrombotic syndrome [8]. Fifty milligrams of r-tPA was applied through the catheter in the first 72 hours. After the first cycle of thrombolysis we used an abdominal $4 \mathrm{MHz}$ convex probe to assess the patency of pelvic veins. We observed a partially dissolved thrombus in the left common femoral vein (CFV), external iliac (EIV) and common iliac vein (CIV). Those veins were compressible, but no flow was detected by Doppler ultrasound. Using ultrasound guidance, we assessed the patency of those veins with a 0.035 -inch J wire, but progression stopped at the level of common iliac vein junction. To define the cause of obstruction, we decided to do an abdominal contrast CT, which showed complete IVCA. Veins from the right lower limb drained through the retroperitoneal venous pathways with well-developed and dilated hemiazygos and azygos vein, paravertebral and even intraspinal veins. The right iliac veins were patent, but no contrast outflow from the left iliac and femoral veins was detected.

After realizing the anomalous venous anatomy of our patient, we performed venous digital subtraction angiography through a 4 Fr sheath inserted into the left GSV. We observed occluded outflow from the common iliac vein on the left side and partially opened CFV, EIV and CIV (Figure 1A,1B) from where the blood drained through suprapubic vein into the right VFC and through the right lateral veins directly into the retroperitoneal venous pathways. The thrombus in those veins dissolved almost completely with thrombolysis. We also observed a conical occlusion of the left paravertebral vein at the L4 spinal level. We recanalized this occlusion with 0.025 -inch hydrophilic straight wire (Terrumo). A hydrophilic Terrumo catheter was placed over the wire through the occlusion in the left paravertebral vein and over azygos vein into the right atrium. After we confirmed that hemiazygos and azygos veins were patent, we inserted a harder 0.035 -inch wire (Amplatz $\left.\mathrm{B}^{\circledR} \mathrm{J}\right)$. We performed balloon dilatation (using 5 and $6 \mathrm{~mm}$ balloon) (Figure 2A, $2 \mathrm{~B}, 2 \mathrm{C}$ ) of the occluded part of the paravertebral vein, during which the patient experienced mild back pain on the left side. Because of residual thrombus adherent to the vascular wall, we decided to continue with thrombolysis (through a sheath in GSV) for additional 16 hours (total dose $10 \mathrm{mg}$ ).

After the last cycle of thrombolysis, Doppler ultrasound showed good patency of the left iliac veins, but the venous flow was weak due to low distal filling of the left iliac veins.

Three days later, control venography was performed. The thrombus in the left pelvic veins completely dissolved with thrombolysis. The previously dilated segment of paravertebral vein was still relatively stenosed, so we decided to further dilate this segment with 7 and 8 $\mathrm{mm}$ balloon. The dilatation was successful with excellent results and a satisfying venous outflow from the pelvic and abdominal region.

Because of persistent thrombosis of the left superficial (SFV) and left deep femoral vein (DFV), venous outflow from the left leg was still obstructed. Our attempt to recanalize the vein retrorogradelly through an existing sheath in the GSV was unsuccessful. Therefore, an additional $4 \mathrm{Fr}$ sheath was inserted under ultrasound guidance anterorogradelly into the distal part of the left SFV (popliteal region). Through this sheath, additional local thrombolysis was performed (total time 24 hours, total dose $10 \mathrm{mg}$ ).

Two days after the final thrombolysis, the final venography was performed through the catheter inserted in the popliteal vein. All the deep leg veins proximally to the popliteal vein were completely patent, along with the left GSV, the left iliac veins and the left paravertebral veins (Figure 3A, 3B, 3C, 3D).

Venous catheters were gradually removed, and the patient was discharged with a therapeutic dose of nadroparine for one month, with factor Xa activity monitored regularly. Because of lack of experience in patients with DVT and IVCA, the use of LMWH was considered a safer alternative in the early phase, rather than the new oral anticoagulant drugs.

One month after hospital discharge, complete patency of the deep leg veins, with minimal non-occlusive adherent thrombosis of the left posterior tibial vein was observed with Doppler ultrasound. The venous flow was normal and respiratory modulated. The GSV and SSV were competent, except for a minimal SFJ insufficiency. We decided to change anticoagulant therapy with nadroparin for new oral anticoagulant drug (rivaroxaban $20 \mathrm{mg}$ o.d.).

Six months after the first hospitalization, magnetic resonance angiography (MRA) showed completely absent infrarenal and suprarenal part of IVC (except for the intra- and suprahepatic part). Venous blood from the lower extremities drained through external iliac veins to paravertebral plexus and on through azygos and hemiazygos vein to superior vena cava. MRA showed good patency of the dilated

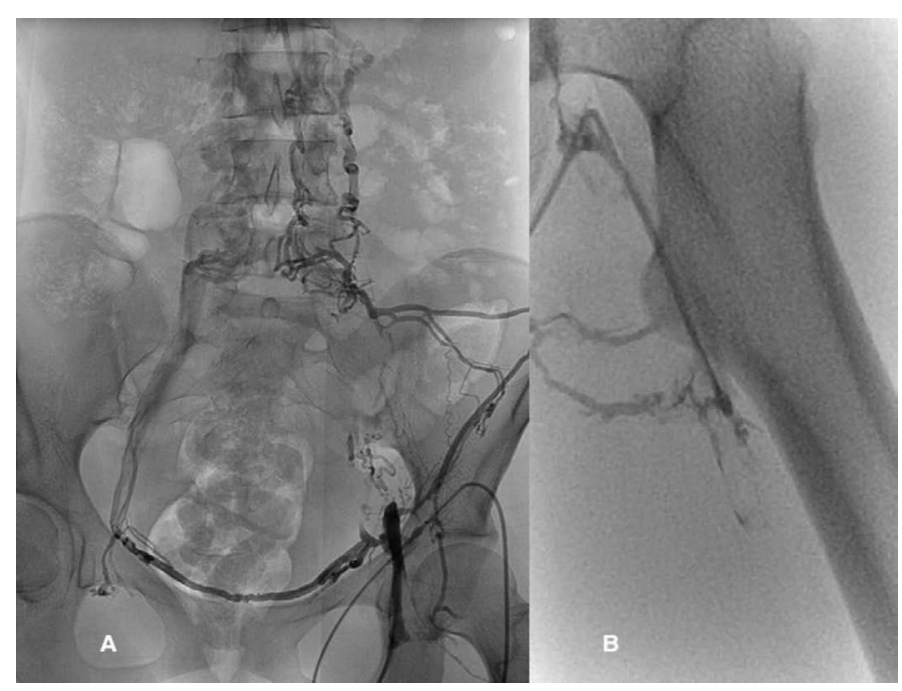

Figure 1A. Pelvic vein occlusion; 1B. Left femoral vein occlusion.

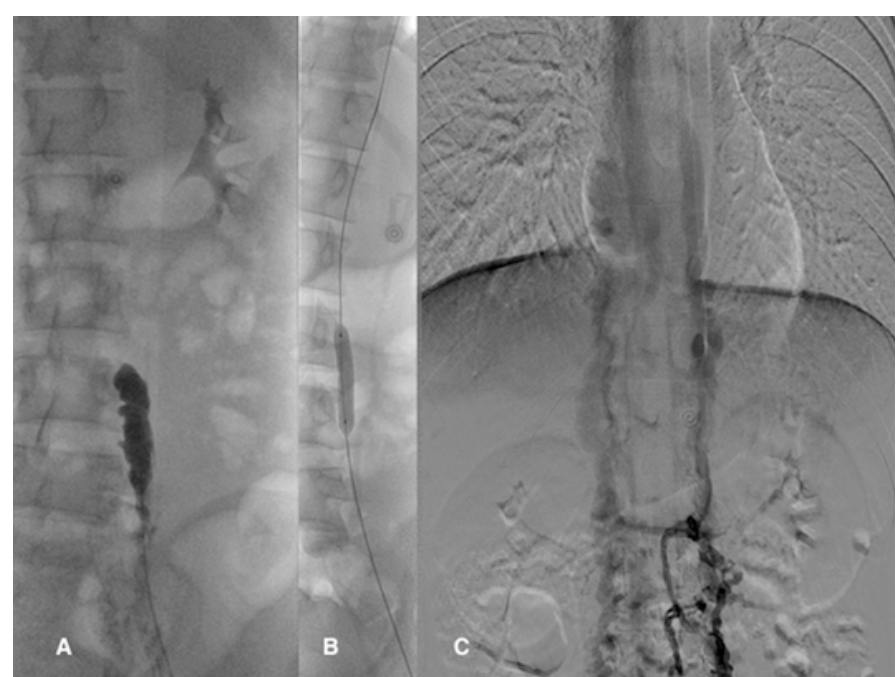

Figure 2A. Paravertebral occlusion; 2B. Paravertebral PTA; 2C. Post-PTA result. 


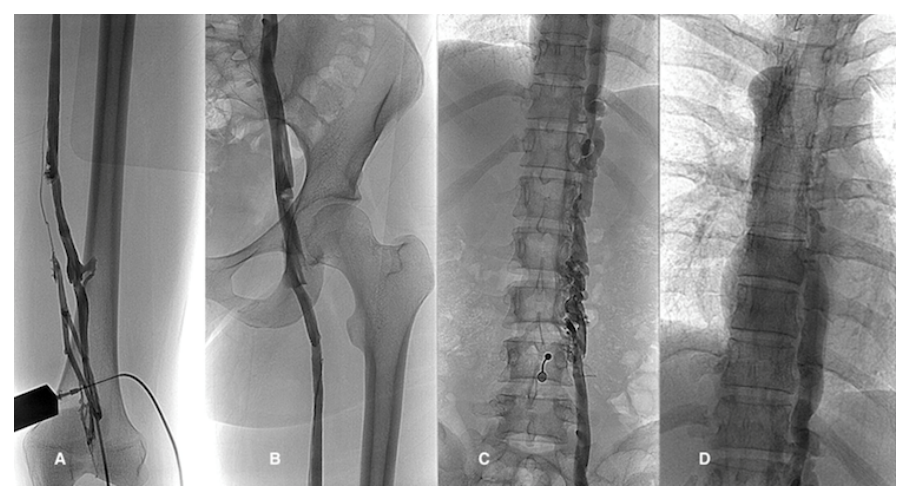

Figure 3A, 3B. Final venogram of femoral veins; 3C, 3D. Final venogram of paravertebral veins.

left paravertebral plexus. Two years after presentation to our hospital (September 2016), a control MRA showed the same results - deep and superficial leg veins and pelvic veins were completely patent along with the dilated paravertebral vein (Figure 4A, 4B). The patient has no residual symptoms - there is no residual leg swelling with comparable circumference of both legs (Figure 5).

\section{Discussion}

IVCA is a rare developmental abnormality (0.005-1\%) [1]. The least common is total IVCA, segmental IVCA or inferior vena cava hypoplasia are more common.

Etiology is still unknown but theory of embryonal dysgenesis and intrauterine or perinatal thrombosis of IVC are postulated [6, 9-11]. IVCA must be differentiated from IVC congenital membranes [12].

The most common presentation of IVCA is uni- or bilateral DVT in patients aged 20-30 years, more commonly in men [1-5]. The trigger is usually physical exercise and the most common complaint back pain [6,13-15]. Diagnostic criteria for IVCA is typical absence of VCI on MRA and typical collateral venous pattern.

Less than 100 cases of IVCA have been described in the english literature [5]. These patients have been treated very differently: from short to long-term anticoagulant treatment, to thrombolysis, extended angioplasty and even surgical bypass. Long-term anticoagulant treatment and avoidance of hormonal contraceptive/substitution treatment is most commonly recommended but there are no controlled randomized studies to confirm this treatment [16].

Our patient was at first treated with LMWH, but the thrombosis progressed. Proximal DVT (LET class III-IV) are generally associated with poorer outcome $[8,17]$ with high incidence of post-thrombotic syndrome (PTS). Because anticoagulation alone does not reduce the overall thrombus burden following DVT, our experience has shown that proximal DVT should not be treated with anticoagulation alone $[8,18]$. Proximal DVT treated with catheter-directed thrombolysis, on the other hand, has been associated with a very low rate of postthrombotic syndrome (PTS) $[8,19]$.

Slow low-dose regional thrombolysis has showed to be as effective as quick high-dose thrombolysis regimen and much safer in treatment of proximal DVT and in patients with IVCA [20] with no serious complications and good mid-term results.

We decided to use our own thrombolysis protocol of local-longlow dose r-tPA infusion. Because of only partially successful flow reestablishment, we used a stepped approach: CTA, classic venography with endovascular treatment, followed by low-dose thrombolysis through GSV and optionally later (after conversion from I-IV to I-II LET class) popliteal vein. Using this approach, we were able to re-establish venous flow in 14 days with relatively low cumulative thrombolytic dose (70 mg r-tPA in 112 hours) and low-aggressive angioplastic procedures. Excellent short and mid-term results were shown but long-term patency is still unknown.

We suggest using individualized approach based on each patient's anatomy and potential pre-existing conditions (for example IVCA). Hybrid and minimally invasive approach allows us to take advantage of every individual approach and avoid the adverse effects. Extensively prolonged or high dose thrombolysis would have more potential side effects as would the use of aggressive angioplasty with stenting [21]. Both therapeutic methods combined provide the best result with minimal complications.

The described way of treatment is relatively easy to perform and to control in comparison to many other known protocols of local thrombolysis. It is feasible in every hospital with intensive care unit where catheters can be placed in the peripheral veins under ultrasound guidance. Endovascular treatment is feasible in hospitals with wellequipped interventional radiology unit and experienced interventional radiologists. Because endovascular treatment is not possible in our general hospital, we collaborated with Clinical Institute of Radiology at University Medical Centre Ljubljana. The final clinical and angiographic outcome is the result of excellent cooperation of two departments from neighbouring hospitals.

\section{Conclusions}

We propose that all patients, especially younger men, with DVT proximally to SFJ and without signs of pulmonary thromboembolism, should have abdominal and pelvic CTA or MRA diagnostics to exclude IVCA.

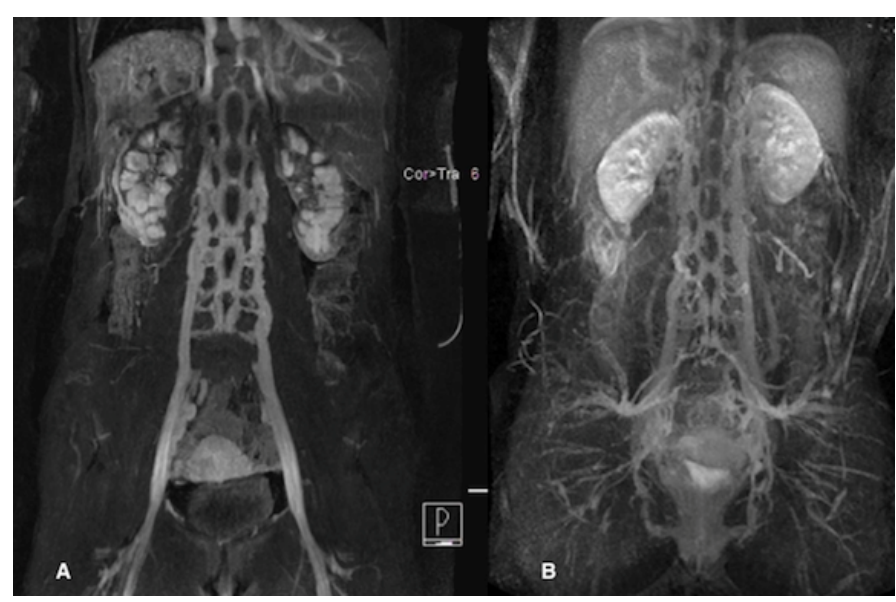

Figure 4A. Control MRA after 6 months; 4B. Control MRA after 2 years.

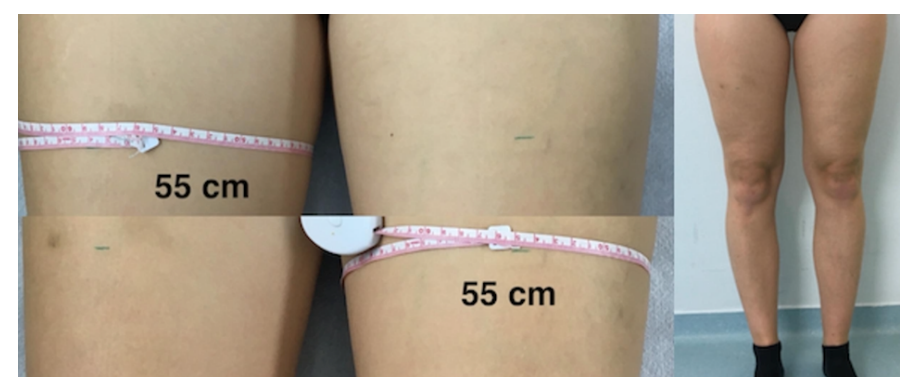

Figure 5. Result - Comparable circumference of right and left thigh. 
When proximal DVT is diagnosed, treatment with LMWH is not sufficient and we have to use other treatment options or combinations of them, based on every case individually. Local thrombolysis with $\mathrm{r}$-tPA in combination with additional angioplasty is worth considering. We suggest using minimally invasive approaches first.

We have successfully combined thrombolysis and interventional procedures in our patient. In addition to thrombolysis, we used minimally invasive approach with only dilatation of the occluded segment in two sessions and no stenting. In the case of DVT recurrence and the occlusion of the ascending lumbar vein, stenting would be necessary. In two years of follow up, the outcome is excellent - the dilated segment is patent, along with all limb veins.

\section{Acknowledgements}

We would like to thank Clinical Institute of Radiology, University Medical Centre Ljubljana, for kind cooperation.

\section{Funding}

There were no sources of funding.

\section{Conflict of interest}

No conflict of interest.

\section{References}

1. Sneed D, Hamdallah I, Sardi A (2005) Absence of the retrohepatic inferior vena cava: what the surgeon should know. Am Surg 71: 502-504. [Crossref]

2. Yun SS, Kim J, Kim KH, Sung GY, Lee DS et al., (2004) Deep venous thrombosis caused by congenital absence of inferior vena cava, combined with hyperhomocysteinemia. Ann Vasc Surg 18: 124-129. [Crossref]

3. Minniti S, Visentini S, Procacci C (2002) Congenital anomalies of the venae cavae: embryological origin, imaging features and report of three new variants. Eur Radiol 12: 2040-2055.

4. Dean SM, Tytle TL (2006) Acute right lower extremity iliofemoral deep venous thrombosis secondary to an anomalous inferior vena cava: a report of two cases. Vasc Med 11: 165-169.

5. D'Aloia A, Faggiano P, Fiorina C, Vizzardi E, Bontempi L et al., (2003) Absence of inferior vena cava as a rare cause of deep venous thrombosis complicated by liver and lung embolism. Int J Cardiol 88: 327-329. [Crossref]

6. Lambert M, Marboeuf P, Midulla M, Trillot N, Beregi JP et al., (2010) Inferior vena cava agenesis and deep vein thrombosis: 10 patients and review of the literature. Vasc Med 15: 451-459. [Crossref]
7. Foegh P, Jensen LP, Klitfod L, Broholm R, Baekgaard N (2017) Factor associated with long-term outcome in 191 patients with ilio-femoral DVT treated with catheterdirected thrombolysis. Eur J Vasc Endovasc Surg S1078-5884:30652-30659 (e-pub ahead of print).

8. Strijkers RH, Arnoldussen CW, Wittens CH (2015) Validation of the LET classification. Phlebology 30: 14-19. [Crossref]

9. Gayer G, Luboshitz J, Hertz M, Zissin R, Thaler M et al., (2003) Congenital anomalies of the inferior vena cava revealed on CT in patients with deep vein thrombosis. AJR Am J Roentgenol 180: 729-732.

10. Obernosterer A1, Aschauer M, Schnedl W, Lipp RW (2002) Anomalies of the inferior vena cava in patients with iliac venous thrombosis. Ann Intern Med 136: 37-41. [Crossref]

11. Ramanathan T, Hughes TM, Richardson AJ (2001) Perinatal inferior vena cava thrombosis and absence of the infrarenal inferior vena cava. J Vasc Surg 33: 1097 1099. [Crossref]

12. Ruggeri M, Tosetto A, Castaman G, Rodeghiero F (2001) Congenital absence of the inferior vena cava: a rare risk factor for idiopathic deep-vein thrombosis. Lancet 357 : 441. [Crossref]

13. Evanchuk DM, Von Gehr A, Zehnder JL (2008) Superficial venous thrombosis associated with congenital absence of the inferior vena cava and previous episode of deep venous thrombosis. Am J Hematol 83: 250-252.

14. Yigit H, Yagmurlu B, Yigit N, Fitoz S, Kosar P (2006) Low back pain as the initial symptom of inferior vena cava agenesis. AJNR Am J Neuroradiol 27: 593-595. [Crossref]

15. Dudeck O, Zeile M, Poellinger A, Kluhs L, Ludwig WD et al., (2007) Epidural venous enlargements presenting with intractable lower back pain and sciatica in a patient with absence of the infrarenal inferior vena cava and bilateral deep venous thrombosis. Spine (Phila Pa 1976) 32: E688-691. [Crossref]

16. Riera-Mestre A, Romera A, Fernandez A, Corbela X (2014) Long-term follow-up after anticoagulant treatment withdrawal in patients with deep venous thrombosis and inferior vena?cava agenesis. Eur J Int Med 25: e113-114.

17. Kahn SR, Shrier I, Julian JA, Ducruet T, Arsenault L et al., (2008) Determinants and time course of the postthrombotic syndrome after acute deep venous thrombosis. Ann Intern Med 149: 698-707.

18. Bækgaard N (2014) Benefit of catheter-directed thrombolysis for acute iliofemoral DVT: myth or reality? Eur J Vasc Endovasc Surg 48: 361-362. [Crossref]

19. Broholm R, Sillesen H, Damsgaard MT, Jorgensen M, Just S et al., (2011) Postthrombotic syndrome and quality of life in patients with ileofemoral venous thrombosis treated with catheter-directed thrombolysis. J Vasc Surg 54: 18S-25S.

20. Broholm R, Jorgensen M, Just S, Jensen LP, Baekgaard N (2011) Acute ileofemoral venous thrombosis in patients with atresia of the inferior vena cava can be treated successfully with catheter-directed thrombolysis. J Vasc Interv Radiol 22: 801-805.

21. Healey CT, Halin N, Iafrati M (2006) Endovascular stenting of ascending lumbar veins for refractory inferior vena cava occlusion. $J$ Vasc Surg 44: 879-881. [Crossref]

Copyright: $\bigcirc 2017$ Leskovar B. This is an open-access article distributed under the terms of the Creative Commons Attribution License, which permits unrestricted use, distribution, and reproduction in any medium, provided the original author and source are credited. 